\title{
Apprivoiser les météores : les baromètres imaginaires de Marie Gevers
}

\author{
Domesticating the seasons: \\ Marie Gevers's fictitious barometers
}

\author{
Véronique Jago-Antoine \\ Archives \& Musée de la Littérature \\ veronique.jago@aml-cfwb.be
}

\begin{abstract}
Belgian novelist Marie Gevers lived all her life surrounded by venerable trees alongside a pond, in the gorgeous estate of Missembourg. Her imagination and sensibility were deeply impregnated by the changing of natural elements and seasons, which were enhanced as authentic characters in her literary work. Plaisir des météores (1938) is emblematic of this impregnation, being a personal and poetical rewriting of legendary almanacs, which invigorated less-literate populations in the remote countryside for centuries after the invention of the printing press. Enlightened by this particular tradition and an analysis of the author's creative stylistic devices, season renewal appears as an everlasting dramaturgy, leading us to adapt ourselves to the world's perpetual metamorphosis, in a pure Ode to Joy.
\end{abstract}

Keywords: Marie Gevers, time, weather, almanac, phenomenology, joy

Il en va de certains lieux comme d'un sortilège : charmeurs ou source d'effroi, ils ont à ce point modulé le destin de ceux qui les ont traversés qu'ils s'évoquent irrépressiblement à l'appel de leur nom. Murmurez le nom de Chateaubriand, et se dressent, sombres et froides, les tours du château de Combourg. Évoquez Proust, et le clocher de Combray appelle à vos yeux la fraîcheur juvénile d'un éternel printemps. Giverny a essentialisé la mémoire des nymphéas de Monet.

Vaste demeure abritée de grands arbres, au bord d'un étang, le domaine anversois de Missembourg enchanta Marie Gevers. Elle y passa sa vie entière, comme plus 
tard, son fils Paul Willems. Ce microcosme édénique n'inspira pas seulement l'un des romans les plus vibrants des lettres belges de langue françaises (Madame Orpha, 1933). Il fut en réalité l'écrin radieux d'une œuvre littéraire qui a réservé au « Tempsqui-passe » et au « Temps-qu'il fait » une hospitalité jusque-là inédite. Refusant de les reléguer en fond de scène, tels les éléments décoratifs d'un théâtre de verdure romantique ou pittoresque, l'auteure les a promus tels des protagonistes à part entière d'une vaste dramaturgie cosmique et comme autant d'actants déterminants de nos existences singulières. On pourrait s'en étonner : parler de la pluie et du beau temps, n'est-ce pas, dit-on, entrer dans la ritournelle des propos sans substance, des conversations creuses, bref faire tourner à vide le langage ?

L'almanach de Plaisir des météores ou le livre des douze mois ${ }^{1}$, publié en 1938 au moment même de la réception de Marie Gevers à l'Académie royale de Langue et de Littérature françaises, offre à ce poncif le démenti le plus savant et le plus exquisément sensuel. Non seulement parce qu'il n'est de fragment de son univers baigné d'eau, d'azur et ourlé de verdure, dont l'auteure n'ait souhaité tisser les sons, les parfums, les couleurs ; ordonner les rythmes ; ravauder les souvenirs ; pour transformer en texte sa texture sensuelle, en épopée ses météores, et permettre à ses habitants autant qu'à ses hôtes - le lecteur y étant convié comme tel - de trouver place en son histoire, et d'évaser celle-ci vers le monde. Mais parce que s'y révèle à quel point les " sortilèges » météoriques emblématiques de l'œuvre geversienne procèdent moins d'un miracle propre au lieu qui les inspire, que d'une quête de sens volontariste, soutenue autant par la rigueur de l'esprit que par l'alerte des sens, par la voix des livres autant que par celle du jardin. Passionné de botanique, Florent Gevers, le père de Marie, en avait notamment posé les bases dès son installation dans sa demeure campagnarde, en truffant d'annotations autographes le recueil d'éphémérides relatives aux phénomènes naturels, qu'il avait acquis aussitôt. Ce Mémorial du Naturaliste et du Cultivateur, qu'il enrichit sa vie durant d'observations minutieuses ${ }^{2}$, ne cessa d'être étoffé par ses descendants, et fut la source même de la réécriture de la tradition almanachienne déployée dans Plaisir des météores avec une sensualité et une imagination jubilatoires.

\section{PLAISIR DES ALMANACHS}

L'origine de cette tradition qui fit florès dès le XVII ${ }^{\text {ème }}$ siècle en marge des réseaux de la librairie traditionnelle, mérite d'être rappelée car elle fascina à ce point Marie

\footnotetext{
${ }^{1}$ Gevers, 1938. Dernière réédition en 2020 (Bruxelles, Espace Nord). Notre pagination se réfère à cette édition dont nous avons signé la postface, inspiratrice du présent article.

${ }^{2}$ Morren, De Vos, 1872. L'exemplaire de la bibliothèque de Missembourg a été relié de cuir en deux charmants volumes à l'initiative de Paul Willems. Il est archivé sous la cote ML 13129 aux Archives \& Musée de la Littérature (Bruxelles), où sont conservés les Fonds d'archives de Marie Gevers et de Paul Willems.
} 
Gevers que celle-ci y référa explicitement deux autres de ses livres, parus aux bornes opposées de son œuvre : en 1930, l'Almanach perpétuel des jeux d'enfants, un recueil de poèmes illustré par l'artiste flamand Félix Timmermans et confié aux soins experts de l'imprimeur anversois J-E Buschmann, et l'Almanach perpétuel des fruits offert aux signes du zodiaque, publié, à Anvers toujours, à la Librairie des arts, en 1965. Confectionnés à vil prix et sans grand soin par des imprimeurs spécialisés dans la littérature de colportage, diffusés par les camelots à travers les campagnes ou proposés sur les étals des merciers parmi les boutons, les fils et les rubans, les almanachs originels, petits livres éphémères " que la même année voyait naître et mourir » ${ }^{3}$ firent jusqu'au cœur même du $\mathrm{XX}^{\mathrm{eme}}$ siècle le bonheur des populations rurales illettrées ou lisant peu.

Entre ces pages de petits formats, troussées telles de naïves encyclopédies de poche, se pressaient avec fantaisie " les maîtres du temps » (calendriers des saints, des lunaisons, des fêtes, des marchés et des kermesses), une kyrielle de rubriques instructives courant des recettes et astuces ménagères aux comptines et dictons, et le plus attendu, sinon l'essentiel : des « pronostications » météorologiques et astrologiques pour chacun des mois de l'année à venir, avec l'indication des jours favorables pour les travaux des champs, l'entretien du bétail ou les déplacements. Biner, sarcler, saigner ou médicamenter, nulle pratique qui ne fût régie par les caprices du temps qu'il fait et le cycle inexorable du temps qui passe, les deux despotes dont Marie Gevers allait ennoblir la tyrannie d'une majuscule pour mieux les apprivoiser.

On réalise peu l'immense succès commercial de ces publications familières, popularisées par leur prix modique, leur format réduit et leurs prédictions racoleuses, des atouts qui en firent la forme de livre la plus répandue à la fin du XVIII ${ }^{\mathrm{e} m e}$ siècle, avant même que les progrès de l'alphabétisation et les innovations techniques apportés à l'imprimerie par l'industrialisation ne chiffrent par millions leur essor à travers l'Europe.

Publié à l'initiative de La Belgique Horticole, une revue savante et richement illustrée connectée à l'Observatoire royal de Belgique et à l'Académie thérésienne, le Mémorial du Naturaliste qui fit le bonheur des hôtes de Missembourg tranche évidemment sur la production des almanachs traditionnels, édifiants certes mais fantaisistes et périssables : le collationnement scrupuleux des données ne s'y égare jamais de façon ludique. Toutefois, la considération réservée par ces savants aux données non scientifiques, telles l'influence du Zodiaque, les légendes et les dictons ne manqua pas d'infléchir l'éveil intellectuel et imaginaire de la jeune écrivaine de Missembourg. Ne légitimait-elle pas l'intérêt réservé par sa mère aux traditions orales et aux savoirs non livresques ? ${ }^{4}$ L'amitié avec le poète Max Elskamp, féru de folklore et d'arts populaires, renchérira en ce sens 5 .

\footnotetext{
${ }^{3}$ Baubérot, 2018, p. 2.

${ }^{4}$ Gevers, 1959 , pp. 37-47.

${ }^{5}$ Leurs chaleureux échanges épistolaires en témoignent ( $c f$. notamment les lettres du Fonds Elskamp enregistrées sous les cotes AML FS12 154/137 et 140).
} 


\section{ALMANACHS ET POÉSIE}

L'évocation d'Elskamp nous rappelle d'évidence que Marie Gevers n'était ni la seule ni la première à trouver des ressources poétiques dans la simplicité populaire des almanachs. Émile Verhaeren, lui-même, son parrain en poésie, n'avait-il pas cédé à leur charme en dédiant aux douze mois de l'année un exquis « cahier de vers » qui mariait ses métaphores aux arabesques de Théo Van Rysselberghe ? L'impression de cet Almanach, paru en 1895 chez l'éditeur bruxellois Dietrich \& Co, avait été réalisée dans un modeste format carré qui rappelait les petits volumes de colportage mais se déclinait, avec un art tout bibliophilique, en quatre versions de couleurs différentes, comme pour accorder le délicat ouvrage au quatuor des saisons.

Quand vient alors son tour de renouveler le genre pour la collection des Livres de nature, dirigée chez Stock par Jacques Delamain - une collection scrupuleusement documentée mais visant à « la connaissance lyrique du monde »-l'écrivaine fait plus que poursuivre filialement les annotations du Mémorial, comme allait s'y astreindre aussi, à sa suite, Paul Willems. Sans rien céder à la justesse naturaliste, son propre almanach fomente une révision originale du modèle qui fictionnalise les données arides des éphémérides en rameutant notamment souvenirs personnels et anecdotes paysannes.

\section{ENCHANTER LE MONDE}

Surtout, elle insuffle au livre la puissance éclairante d'une écriture. De l'austère inventaire, elle fait une épopée, et des variations atmosphériques - vents, givre ou arc-en-ciel - les acteurs d'une véritable cosmogonie, soutenue par les « sortilèges » de métaphores synesthésiques et de «mots-fées ». Le « beau mot» de météores n'est-il pas l'un de ses plus savoureux glanages, une trouvaille de poète ? Pas une trouvaille, plutôt une re-trouvaille, s'empresse de préciser l'auteure, dans une préface qui feint de n'emprunter qu'à la profusion méconnue du lexique :

Les météores? On a pris l'habitude de ne nommer météores que les astres errants, les étoiles filantes ou la foudre. Or, tous les phénomènes qui se passent dans l'atmosphère répondent à ce beau nom. La grêle, le brouillard et les pétales de la rose des vents sont des météores, ainsi que le givre, le grésil et le dégel, l'arc-en-ciel et le halo lunaire, et, aussi, les silencieux éclairs de chaleur où se libère l'angoisse des nuits de juillet ; météores enfin le rougeoiement des couchants et les lueurs vertes de l'aube (p. 8).

Marie Gevers se veut objective. Elle ne crée rien : il lui suffit d'exercer ses cinq sens. Elle n'invente rien : il s'agit simplement de déplier les mots. Son geste, cependant, accomplit bien davantage qu'une pure restauration. Ne se joue-t-elle pas 
de nous quand feignant l'énumération académique des propriétés de son objet, elle s'égare ingénument jusqu'aux « pétales de la rose » des vents ; lorsqu'elle soumet les phénomènes de la nature à une frémissante projection d'affects (de «l'angoisse des nuits de juillets » à « l'énervement des giboulées ») ; et que s'agissant de rendre aux météores « leur véritable nom », elle personnalise la Pluie et le Vent d'une majuscule, et convoque l' "Anti-mai » parmi les « demi-dieux ailés » de son épopée cosmologique ? Rétive aux catégories, son ambition tout à la fois naturaliste, poétique et pluri-sensorielle se résume d'un trait audacieux :

Ayant redonné leur véritable nom à tous ces demi-dieux ailés, il faudra aussi que nous rendions à nos sens émoussés leur subtilité première. Dès lors, tous les plaisirs des météores nous deviendront accessibles (p. 8).

\section{DE L'ALMANACH À L'ART POÉTIQUE : RÉINVENTER LES MÉTÉORES}

Dire pour mieux ressentir, nommer pour éprouver : les « mots-fées » que l'auteure collationne ou confectionne méritent ce qualificatif parce qu'ils ensorcellent notre existence en élargissant le spectre de notre appréhension du monde ${ }^{6}$. Égarer l'imagination parmi les nomenclatures naturalistes, vagabonder par-delà les frontières linguistiques, sauter entre les niveaux de langue ou expérimenter au hasard des qualificatifs picorés dans le dictionnaire offriront donc autant de voies transversales pour « se déplacer mentalement » dans un monde rendu à sa vastitude, non cloisonnée et sans borne. Alors que d'insolites expériences physiques concourent à l'enchantement : «Comment donc est l'air dont on a soustrait toute buée? ? (p. 22), un discret portrait anonyme glissé entre les pages du calendrier geversien nous en divulgue le ressort :

Je me souviens de toiles où l'artiste n'osa même pas attendre que la neige fût tombée. Il l'a peinte dans sa chute tourbillonnante, sur un fond presque indistinct de bois ou de maison : il l'a représentée, non comme un objet, mais dans son activité de météore (pp. 188-189).

Si l'on reconnaît aisément entre les lignes la silhouette du peintre Frans Willems, époux de Marie Gevers, nul doute qu'il s'y formule davantage qu'un délicat hommage amoureux. Une si ardente complicité d'art s'élucide entre ces lignes que la quête picturale de l'un emblématise le dessein poétique de l'autre. « Courons au jar-

${ }^{6}$ De la « marguerite exfoliée » d'Apollinaire (Alcools, « Voie lactée I », v. 28, 1913) au « Trique-madame » (l'« orpin blanc", sedum album) campinois, sa collecte est éclectique, même si la pureté du simple « azur » compte parmi ses favoris. 
din, dans les champs, sous la pluie », nous enjoint souvent Marie Gevers avec l'empressement jubilatoire de son mari. La subtilité de sa palette poétique est redevable à cette hâte captieuse d'aquarelliste : les rondeurs « bleu-paon » de juin (p. 92), les «tons de nuées imbibées d'aubes » des fleurs d'ormes (p. 28), ou telle « averse couleur de pervenche » (p. 68) ne séduisent pas seulement par leur exquise délicatesse ; ils conduisent notre imagination sensorielle vers des états élémentaires hybrides, parfaitement en phase avec la pulsation génératrice du monde?

Libérée par la fluidité de l'aquarelle, l'imagination poétique de Plaisir des météores s'aventure à rêver l'élan vers le ciel - «l'activité de météores 》- de toutes les composantes du vivant. Au printemps, la sève qui bleuit les arbres mêle « les chevelures des saules à l'azur faible des matins » (p. 66), « le chant du rossignol se confond avec la lumière lunaire » (p. 80), « le coquelicot n'est qu'un cri » vers le soleil (p. 84), tandis que, gonflé comme un essaim, le poirier sur le point de fleurir prend «sa valeur complète d'insecte ailé » (p. 60). Cette " météorisation » cosmologique subjugue lorsque son aire s'élargit aux gestes mêmes des humains : « Non je ne me souviens plus de nos jeux. Mais je me rappelle le souffle de nos jeux » (p. 88). Cette notation bouleversante ne mérite pas seulement d'être épinglée pour sa hardiesse à faire de la mémoire elle-même une productrice de météores. Véritable coup de force poétique, elle atteste que les licences imaginaires de Marie Gevers n'émanent pas d'une curiosité de pure spectatrice. S'y formule un véritable credo phénoménologique.

\section{CONNAITTRE POUR CO-NAÎTRE}

Goûter les infinies saveurs de la pluie - en particulier celle de la goutte d'eau de février sur la tige du perce-neige « indéfinissable, un peu acidulée, un peu amère, timide et comme étonnée » (p. 30)-, discerner les changements de la sonorité de l'air (p. 49) ou les parfums du dégel (p. 24) n'édifie pas un simple catalogue de sensations. C'est une immersion corps et âme dans l'épaisseur sensorielle de l'instant.

Pendant les nuits de gel, le ciel et la terre n'appartiennent qu'aux astres [...] Plus vous regarderez [les étoiles] plus il en viendra, et si vous persistez, elles vous attaqueront l'âme. Il est temps de les quitter (p. 11).

On mesure l'écart entre la collecte empirique du naturaliste, scrupuleuse et distanciée, et l'engagement de ce « je » poétique ardent, réceptif à la motricité particulière des qualités sensibles : « Le vent du nord fait vibrer toutes les cordes de l'air. L'azur

\footnotetext{
${ }^{7}$ Notons ici que de charmants carnets de dessins et plusieurs aquarelles conservés dans le Fonds Marie Gevers témoignent d'expériences plasticiennes qui prendront fin lorsque la voix des mots imposera définitivement ses tonalités originales. Plusieurs œuvres de Frans Willems figurent également dans les collections des Archives \& Musée de la Littérature.
} 
est si tendu qu'il sonne, puis casse (p. 24)». Un « je » dont les injonctions fougueuses, impératives, exclamatives n'ont de cesse de se pluraliser en " nous » pour secouer notre indolence distraite. Marie Gevers n'écrit pas pour des regardeurs contemplatifs ; elle mobilise son lecteur par des protocoles de guet qui le rendent si perméable à l'énergie transformiste du monde qu'il en devient le baromètre ou plutôt la rose des vents:

Et voici que soudain tout l'espace floconne, danse, fleurit, et que toute la lumière du ciel vient baiser la terre [...].

Tenons-nous au centre de tout, comme si nous étions la rose des vents, immobile au commencement du monde (p. 186).

Merleau-Ponty ou Bachelard, des compagnons de pensée pour Marie Gevers, n’auraient pu concevoir plus subtile métaphore du « corps phénoménal » : un corps synchronisé avec ce qui l'entoure et modelé par son inscription dans l'espace et dans le temps.

\section{L'INIFNI AU SINGULIER}

«Commencé du plus loin qu'il m’en souvienne, écrit en 1938, sera jusqu’à ma mort toute ma vie (p. 192) » signe Marie Gevers, au terme de Plaisir des météores. La rature autographe de son manuscrit ${ }^{8}$ concorde avec l'élan vitaliste dont elle se veut la traductrice tandis qu'un éloquent da capo le relance à l'infini, telle la ritournelle enfantine en épilogue de Madame Orpha:

Ainsi parlait Eve /

Ainsi parlait Eve /

Ainsi parlent les filles d'Eve'.

Cette relance pourrait toutefois donner l'impression d'enliser la destinée humaine dans le cycle immuable de la répétition. La péremption des almanachs annuels traditionnels ouvrait, en effet, un espace virtuel au changement : si prévisible que puisse être l'année future, elle ne serait en tout cas pas identique. Les emblèmes immuables définis ici s'appliquent, au contraire, à déceler dans chaque mois qui s'achève la présence en puissance de celui à venir : l'idée de fenaison court déjà dans les blés de

${ }^{8}$ Dossier d'archives « Plaisir des météores » conservé dans le Fonds Marie Gevers des Archives \& Musée de la Littérature (Bruxelles), sous la cote FS55 1/15. Le chercheur trouvera dans cet important dossier des manuscrits autographes et des tapuscrits annotés, plusieurs prépublications en revues, des correspondances et de très nombreux extraits de la presse belge et étrangère. Dorénavant AML, FS55 1/15.

${ }^{9}$ Gevers, 1933, rééd. 1992, p. 179. 
juin et la belle assertivité des verbes au futur promet que tout, et toujours, sera « en équilibre », bien « à sa place» (p. 150).

Une astucieuse dialectique du singulier et du pluriel entretenue de diverses manières au fil des pages brise cependant ce cercle potentiellement oppressant. L'élargissement du spectre météorique à des phénomènes aussi inattendus que le son de l'angelus « qui donne aux choses une forme de cloche » (p. 147), l'extension du champ exploratoire depuis les profondeurs de l'étang jusqu'aux nuées en sautant les haies jusqu'aux champs, démultiplient les perspectives d'émerveillement saisonnières tandis que l'hybridation des règnes ne cesse de désarçonner nos savoirs trop clivés : ne prête-telle pas des nageoires au lichen (p. 87) et une torpeur bovine à la canicule estivale?

Alors le soleil s'attaque à la masse des eaux dont luisent nos plaines. Il s'appuie à tous ces miroirs mouvants ou immobiles, et il boit, comme du bétail à l'abreuvoir (p. 115).

Le choix des évocations singulières, qui personnalisent $l e$ hêtre ou le séneçon, agit dans le même sens. Dans le monde geversien, l'élan du vivant ne connaît pas le pluriel : il s'énonce au singulier, comme autant de micro-volontés propres, mais unanimes. On observera que les différents états des manuscrits renchérissent sur ce « grand œuvre » cosmique par un faisceau de retouches qui pourraient sembler de détail si ne les animait un même scrupule visant à intentionnaliser les évocations : ainsi de l'inquiétude portant sur « te signe » de l'arrivée du printemps qu'une rature infléchit significativement vers la question : « où donc se montrera[-t-il] ?»» (p. 74. Nous soulignons).

\section{UNE ODE À LA JOIE}

Sans doute cette projection anthropomorphique apparaît-t-elle à l'occasion bien naïve, sinon franchement surannée ${ }^{10}$. On peut le concéder. Non sans prêter l'oreille à la dimension légendaire ouverte à l'initiale du récit par l'hypothétique « On dirait que le mois de janvier considère les quelques jours de gelée qu'il nous doit comme une dette difficile à payer (p. 9. Nous soulignons) ». Cette formulation, qui semble faire écho à l'immémorial « Il était une fois » des conteurs, invite, en réalité, à consentir à la féerie comme à une composante intrinsèque du réel : « Le sureau est plein d'histoires, tout comme il est plein d'oiseaux (p. 141) ». Le savant Mémorial, Marie Gevers le rappelle, ne formulait-il pas de longue date une requête identique?

\footnotetext{
${ }^{10}$ La correspondance avec l'éditeur révèle la suppression en ce sens du «poème des tulipes » conservé dans le manuscrit original (AML, FS55 1/15/1 et 13). Ajoutons à ces réserves que si les anecdotes paysannes constituent la facette du recueil qui s'est le plus ternie au fil du temps, Jean de Beucken l'avait déjà pressenti dans son compte rendu publié dans La Meuse (12/12/1938 et AML, FS55 1/15/23 (10)).
} 
Les annotations au crayon qui le surchargent sont parfois belles comme un poème, mystérieuses comme une légende [...]. Si notre désir de logique et de preuves y met des points d'interrogation, c'est bien à tort. Il faudrait admettre ces affirmations comme nous admettons le vent, les nuages, les saisons inégales (p. 70).

Admettre, consentir. Ce n'est pas peu d'affirmer que la totalité du travail geversien s'applique à cette posture d'équilibriste :

Nous sommes en train de perdre le soleil, soit mais nous sommes en train de regagner l'eau. Et avec l'eau, les aubes et les soirs imprécis, enveloppés, mobiles ; les jours mouvants, les pans d'averses, suivies de coulées de clarté. Comment avons-nous pu être angoissés par le tournant du solstice [...]? (p. 139)

En suivant hardiment la crête du paradoxe qui reconnaît dans l'impermanence du monde le principe même de son immortalité, les mythologies de Plaisir des météores ne tentent rien d'autre que d'ajuster notre regard à l'énigme insensée du cosmos.

Un bref ex-cursus sous des latitudes étrangères à la quiétude de Missembourg l'illustre d'une ultime façon. Un voyage en Afrique modifie-t-il, à titre exceptionnel, les coordonnées immuables de l'univers geversien ? ${ }^{11}$ Plume à la main en guise de stéthoscope posé sur les météores déconcertants des étendues sub-équatoriales, la voyageuse confesse en toute franchise que « le vertige moral» suscité par « l'air [...] trop puissant, le soleil trop vertical, l'azur trop intense $»^{12}$, est long à se résoudre :

Les mois perdaient leur signification [...] les carrés de fraisiers venus d'Europe portaient perpétuellement et simultanément fleurs et fruits. Comment leur indiquer leur saison? $[\ldots]$

Je me trouvais, tout comme les mots apportés, les fraisiers, les couleurs, désorientée au sens propre du $\operatorname{mot}^{13}$.

Pour endiguer cette déroute des sens et de l'âme, l'écrivaine ne tergiverse guère. Pas d'autre résolution que de remobiliser l'arsenal familier du poète : ajuster scrupuleusement les mots à la versatilité du réel :

Ces mots si exacts en Europe, il allait falloir en user par $2^{\circ} 33^{\prime}$ de latitude sud, sur $29^{\circ} 35^{\prime}$ de longitude est, et 1650 mètres d'altitude. Je m'aperçus vite qu'ils ne convenaient pas aux objets que je leur désignais, et qu'il fallait les assouplir, les dresser à servir les choses nouvelles ${ }^{14}$.

${ }^{11}$ Elle y rejoint à trois reprises sa fille Antoinette Schuermans, épouse d'un ingénieur belge en mission au Rwanda. Deux livres y prendront leur source : Des mille collines aux neuf volcans (1953) et Plaisir des parallèles (1958), tous deux publiés chez Stock.

${ }^{12}$ Gevers, 1958, rééd. 2002, p. 50.

${ }^{13}$ Ibidem, p. 15

${ }^{14}$ Ibidem, pp. 13-14. 
Et fouailler les pouvoirs conjuratoires de l'imagination, jusqu'à susciter, par exemple, la prouesse d'un arc en ciel dans la chaleur zénithale :

Dehors, le soleil avait dévoré toutes les couleurs. Pour être certaine qu'elles n'étaient pas mortes pour de bon, j'ai étendu sur ma couchette un petit fichu bariolé ${ }^{15}$.

La malicieuse simplicité du stratagème et son humble volontarisme sont révélateurs : la poétique météorique de Marie Gevers est une ode à la joie. Pas le « carpe diem » d'Horace, ombré par le pessimisme épicurien, ni la démesure jouisseuse d'un Rabelais. Encore moins un heureux vague à l'âme immotivé. Mais la pure et pleine joie de vivre, célébrée par le philosophe Clément Rosset comme la " force majeure » de celui qui « respire à l'aise [... dans] une existence éphémère, périssable, toujours changeante et désirée comme telle $»^{16}$. Considéré de la sorte, le projet geversien ne vise pas à fuir l'Histoire, ses violences et ses scandales, comme on en a fait quelquefois le reproche à l'auteure : il s'applique seulement à les tenir à la distance nécessaire pour nous permettre de vivifier nos capacités résilientes en captant, par tous nos pores - ceux de l'esprit autant que ceux du corps - l'énigmatique pulsion régénérante de l'univers élémentaire.

La pertinence de cet effort d'adéquation vitaliste au regard de nos inquiétudes actuelles interpelle. Sous l'impulsion de son élan transformiste, les barrières génériques tombent, science et poésie s'enlacent et 《la chair du monde $»^{17}$ prend d'ineffables saveurs insolites. Épeler les joies du réel pour les multiplier et mieux les éprouver. En cet art rayonnant d'habiter poétiquement le devenir versatile de l'existence ne se glisserait-il pas, en définitive, un des secrets de jouvence de l'enchanteresse de Missembourg ${ }^{18}$ ? Nul doute que ses livres vibrants nous soient dédiés en ce sens ${ }^{19}$.

${ }^{15}$ Ibidem, p. 156.

${ }^{16}$ Rosset, 2011, p. 20.

${ }^{17}$ Merleau-Ponty (1988).

${ }^{18}$ Marie Gevers, elle-même, emprunta à Andersen le titre du conte « La femme qui faisait des enchantements » pour faire l'éloge de Max Elskamp (Gevers, 1967, pp. 64-84).

${ }^{19}$ Comme l'énonce la belle dédicace de Plaisir des météores : « Ce livre est dédié / aux habitants des contrées soumises au Gulf-stream » (p. 5). 


\section{BIBLIOGRAPHIE}

Bachelard, G. ( 1992). L'Air et les songes. Paris : PUF.

Baubérot, A. (2018). Prophéties et prédictions astrologiques dans les almanachs populaires du XIXe siècle. In C. Barel-Moisan, A. Déruelle et J.-L. Diaz (Eds.), Le XIXème siècle au futur. Penser, représenter, rêver l'avenir au XIXe siècle. Actes du VIIe Congrès de la SERD. Retrieved from https://serd. hypotheses.org/2038.

Becker, K. (Ed.) (2012). La Pluie et le beau temps dans la littérature française. Paris : Hermann.

Correspondance de Marie Gevers (1986). Choix de lettres annoté par Cynthia Skenazy. Bruxelles : Labor, coll. Archives du Futur.

Dossier d'archives «Plaisir des météores » (FS55 1/15) conservé dans le Fonds Marie Gevers des Archives \& Musée de la Littérature (Bruxelles).

Gevers, M. (1933). Madame Orpha. Paris : Attinger. Rééd. (1992). Bruxelles : Espace Nord.

Gevers, M. (1938). Plaisir des météores ou le livre des douze mois. Paris : Éditions Stock - Delamain et Boutelleau, coll. Les Livres de Nature. Rééd. ( 2020) Bruxelles : Labor.

Gevers, M. (1958). Des mille collines aux neufs volcans. Paris : Éditions Stock. Rééd. (2002). Bruxelles, AML Éditions, Documents pour l'histoire des francophonies.

Gevers, M. (1959). Comment naît une vocation littéraire. Bulletin de l'Académie royale de Langue et de Littérature françaises de Belgique, XXXVI, no. 1, 37-47. Retrieved from https://www.arllfb.be/ bulletin/bulletinsnumerises/bulletin_1959_xxxvii_01.pdf.

Gevers, M. (1967). Souvenirs sur Max Elskamp. Bulletin de l'Académie de Langue et de littérature françaises de Belgique, XLV, no. 2, 64-84. Retrieved from https://www.arllfb.be/bulletin/bulletinsnumerises/bulletin_1967_xlv_02.pdf.

Laensbergh, M. (1978). Almanach supputé sur le méridien de Liège pour 1978. Liège : Vaillant-Carmanne, $335^{\text {ème }}$ année.

Merleau-Ponty, M. (1988). Le Visible et l'invisible. Paris : Gallimard.

Morren, É., De Vos, A. (1872). Mémorial du Naturaliste et du Cultivateur. Liège : La Belgique horticole. Exemplaire de la bibliothèque de Missembourg, enrichi d'annotations autographes et de fragments imprimés (AML, ML 13129).

Petit, N. (1997). L'Éphémère, l'occasionnel et le non-livre à la bibliothèque Sainte-Geneviève : XV'-XVIII siècles. Paris : Klincksieck.

Rosset, C. (2011). La Force majeure. Paris : Éditions de Minuit.

Skenazy, C. (1983). Marie Gevers et la nature. Bruxelles : Palais des Académies. 